\title{
The Influence of Jia Wei Cang Er San on the Postoperative Bacteriology of Chronic Rhinosinusitis: A Randomized, Placebo-Controlled, Double-Blind Study
}

\author{
Rong-San Jiang ${ }^{1,2,3^{*}}$, Kai-Li Liang ${ }^{1,2,3}$, Shang-Heng $\mathrm{Wu}^{1}$, Jen-Fu Lin $^{4}$, Chen-Chen Tsai ${ }^{5}$ \\ ${ }^{1}$ Otolaryngology, Taichung Veterans General Hospital, Taichung City, Taiwan \\ ${ }^{2}$ School of Medicine, Chung-Shan Medical University, Taichung City, Taiwan \\ ${ }^{3}$ Faulty of Medicine, National Yang-Ming Medical University, Taipei City, Taiwan \\ ${ }^{4}$ Pathology and Laboratory Medicine, Taichung Veterans General Hospital, Taichung City, Taiwan \\ ${ }^{5}$ Chinese Medicine, Taichung Veterans General Hospital, Taichung City, Taiwan \\ Email: ${ }^{*}$ rsjiang@vghtc.gov.tw
}

Received February 17, 2013; revised March 18, 2013; accepted March 25, 2013

Copyright (C) 2013 Rong-San Jiang et al. This is an open access article distributed under the Creative Commons Attribution License, which permits unrestricted use, distribution, and reproduction in any medium, provided the original work is properly cited.

\begin{abstract}
Background: Antibiotics have been used routinely for postoperative care in patients with chronic rhinosinusitis (CRS). However, increased bacterial growth was found after antibiotic treatment. In traditional Chinese medicine, Jia Wei Cang Er San has been used to treat CRS. This study was to investigate the influence of Jia Wei Cang Er San on the postoperative bacteriology of CRS. Methods: Ninety-seven CRS patients who underwent functional endoscopic sinus surgery (FESS) were included. They were randomly divided into 3 groups. In the group of Chinese herbal medicine (CHM), patients were given a capsule of Jia Wei Cang Er San tid for 8 weeks and a placebo capsule for amoxicillin q8h for 4 weeks after FESS. In the amoxicillin group, patients were given a capsule of amoxicillin $250 \mathrm{mg}$ q $8 \mathrm{~h}$ for 4 weeks and a placebo capsule for Jia Wei Cang Er San tid for 8 weeks. In the placebo group, patients were given both placebo capsules. Bacterial cultures were performed from bilateral middle meati before FESS, and 8 and 12 weeks after FESS. Results: In the CHM group, bacteria grew in $21(46 \%)$ of 46 specimens pre-operatively, in $23(50 \%)$ specimens 8 weeks and in 17 (37\%) specimens 12 weeks after surgery. In the amoxicillin group, bacteria grew in 15 (28\%) of 54 specimens pre-operatively, in $30(56 \%)$ specimens 8 weeks and in $32(59 \%)$ specimens 12 weeks after surgery. In the placebo group, bacteria were found in 13 (34\%) of 38 specimens pre-operatively, in $16(42 \%)$ specimens 8 weeks and in $12(32 \%)$ specimens 12 weeks after surgery. The rates of bacterial growth did not change by Jia Wei Cang Er San 8 or 12 weeks after surgery, but increased significantly by amoxicillin 8 and 12 weeks after surgery. Conclusion: Our study showed that Jia Wei Cang Er San did not induce bacterial growth after FESS as amoxicillin.
\end{abstract}

Keywords: Amoxicillin; Bacteriology; Chinese Herbal Medicine; Chronic Rhinosinusitis

\section{Introduction}

Functional endoscopic sinus surgery (FESS) has become a standard surgical modality for treating chronic rhinosinusitis (CRS) [1]. Recently, it has been emphasized that postoperative care is very important in the outcome of FESS [2]. Antibiotics are used routinely for post-FESS care [3], because it is generally believed that damaged sinuses are prone to bacterial infection [2]. However, increased bacterial growth was found after antibiotic treatment [4].

Jia Wei Cang Er San which is one of most common

${ }^{*}$ Corresponding author. used Chinese herbal medicines (CHM) to treat rhinosinusitis in Taiwan has been reported to alleviate the nasal symptoms of allergic rhinitis by way of its inhibitory effect on type I anaphylaxis reactions and eosinophil infiltration in the nasal tissues as well as the inhibition of some mediators related to allergic rhinitis $[5,6]$. One of its active components, Angelica dahurica, has been shown to possess antibiotic and cyclooxygenase- 2 inhibitory activities and is used to treat rhinosinusitis [7]. When both antibiotics and CHM were commonly used to treat CRS in Taiwan, we tried to compare the influence of Jia Wei Cang Er San with amoxicillin on the post-FESS bacteriology in CRS patients. 


\section{Materials \& Methods}

A randomized, placebo-controlled, double-blind study was performed to investigate the influence of Jia Wei Cang Er San on the bacteriology after FESS. CRS patients who had poor response to medical treatment and subsequently underwent FESS were included in this study. The diagnosis of CRS was based on a history of rhinosinusitis for more than 12 weeks, the findings of nasal endoscopy, and an examination of CT scans. Any patient who had a history of immunodeficiency or a previous sinus surgery was excluded from the study. Patients who took antibiotics within a week before FESS were also excluded. This study was approved by the Institutional Review Board of Taichung Veterans General Hospital (TCVGHC06215).

On the day before FESS, all patients received endoscopic examination, and endoscopic appearances were quantified on a $0-2$ point scale according to the staging by Lund and Mackay [8]. Endoscopic appearances included the presence of polyps (0: no polyp; 1: polyps confined to the middle meatus; 2: polyps beyond the middle meatus), discharge ( 0 : no discharge; 1 : clear, thin discharge; 2: thick, purulent discharge) and edema. The endoscopic appearances were scored unilaterally.

A swab specimen was taken from the right and left middle meati and sent to the laboratory for aerobic and anaerobic cultures.

After surgery, patients were randomly assigned to 3 groups by a technician. In the CHM group, patients were given a capsule of Jia Wei Cang Er San tid for 8 weeks and a placebo capsule for amoxicillin $\mathrm{q} 8 \mathrm{~h}$ for 4 weeks. A capsule of Jia Wei Cang Er San contains $2 \mathrm{~g}$ of Cang Er San and $1 \mathrm{~g}$ of Yuxingcao. In the amoxicillin group, patients were given a capsule of amoxicillin $250 \mathrm{mg}$ q8h for 4 weeks and a placebo capsule for Jia Wei Cang Er San tid for 8 weeks. In the placebo group, patients were given a placebo capsule for Jia Wei Cang Er San tid for 8 weeks and a placebo capsule for amoxicillin q8h for 4 weeks. During this period, no antihistamine, oral or nasal steroid was prescribed. Nasal irrigation was not performed either.

At 8 weeks after FESS, patients received another endoscopic examination and swab specimens were taken from the right and left middle meati for aerobic and anaerobic cultures. At 12 weeks after FESS, patients received another endoscopic examination again. Swab specimens were also taken from the right and left middle meati for aerobic and anaerobic cultures. The postoperative endoscopic appearances were quantified on a $0-2$ point scale according to the staging method described by Lund and Mackay [8]. Endoscopic appearances included the presence of polyps (0: no polyp; 1: polyps confined to the middle meatus; 2 : polyps beyond the middle meatus), discharge (0: no discharge; 1: clear, thin discharge;
2: thick, purulent discharge), edema, scarring or adhesions and crusting.

The rates of bacterial culture were compared before and after FESS, and among the three groups using the Chi-square test. The endoscopic scores before and after FESS were compared using the Wilcoxon signed rank test. The endoscopic score were compared among the three groups using the Kruskal Wallis test. The bacterial culture rate was calculated by the number of specimens which grew bacteria per total number of specimens. Two-tailed $\mathrm{p}$-values $<0.05$ were considered statistically significant. All computations were performed using the SPSS version 12.0 (SPSS Inc., Chicago, IL, USA).

\section{Results}

There were 97 patients enrolled in this study. Sixty-nine (45 males, 24 females) completed the study with 23 in the CHM group, 27 in the amoxcillin group and 19 in the placebo group. Their ages ranged from 14 to 82 years with a mean of 41.8 years.

Table 1 shows endoscopic scores and prevalence rates of positive bacterial culture before and after FESS in the 3 groups. When endoscopic scores were compared before and after FESS, the scores significantly decreased at 8 weeks after FESS in all 3 groups $(p=0.001$ for CHM group, 0.002 for amoxicillin group and 0.002 for placebo group, respectively) and were lower still at 12 weeks after FESS ( $\mathrm{p} \leq 0.0001$ for CHM group, 0.0001 for amoxicillin group and 0.001 for placebo group, respectively).

Table 1. Endoscopic scores and rates of positive bacterial culture before and after FESS.

\begin{tabular}{|c|c|c|c|c|}
\hline & $\operatorname{CHM}\left(46^{\#}\right)$ & Amoxicillin (54) & Placebo (38) & p-value \\
\hline \multicolumn{5}{|l|}{$\begin{array}{l}\text { Endoscopic } \\
\text { scores }^{\# \#}\end{array}$} \\
\hline Pre-FESS & 3 (2 to 4.25$)$ & $3(2$ to 4$)$ & 3 (2 to 4$)$ & 0.315 \\
\hline $\begin{array}{l}8 \text { weeks after } \\
\text { FESS }\end{array}$ & $2(2$ to 3$)$ & $2(2$ to 3$)$ & $2.5(2$ to 3$)$ & 0.617 \\
\hline p-value ${ }^{*}$ & 0.001 & 0.002 & 0.002 & \\
\hline $\begin{array}{l}12 \text { weeks after } \\
\text { FESS }\end{array}$ & 2 (1 to 3$)$ & $2(2$ to 3$)$ & 2 (2 to 3$)$ & 0.821 \\
\hline$p$-value ${ }^{* *}$ & $<0.0001$ & 0.0001 & 0.001 & \\
\hline \multicolumn{5}{|l|}{$\begin{array}{l}\text { Positive culture } \\
\text { rates }\end{array}$} \\
\hline Pre-FESS & $21 / 46(46 \%)$ & $15 / 54(28 \%)$ & $13 / 38(34 \%)$ & 0.173 \\
\hline $\begin{array}{l}8 \text { weeks after } \\
\text { FESS }\end{array}$ & $23 / 46(50 \%)$ & $30 / 54(56 \%)$ & $16 / 38(42 \%)$ & 0.446 \\
\hline p-value ${ }^{*}$ & 0.835 & 0.006 & 0.637 & \\
\hline $\begin{array}{l}12 \text { weeks } \\
\text { after FESS }\end{array}$ & $17 / 46(37 \%)$ & $32 / 54(59 \%)$ & $12 / 38(32 \%)$ & 0.015 \\
\hline$p$-value ${ }^{* *}$ & 0.525 & 0.002 & 1 & \\
\hline
\end{tabular}

FESS: functional endoscopic sinus surgery; CHM: Chinese herbal medicine; \#Number of sides; "Sides of nasal cavites; "Comparison between pre-FESS and 8 weeks after FESS; ${ }^{* *}$ Comparison between pre-FESS and 12 weeks after FESS. 
The endoscopic scores were not significantly different among the 3 groups either before or after FESS.

The prevalence rates of positive bacterial culture were not significantly decreased after surgery in all 3 groups. In contrast, the prevalence rates of bacterial culture growth increased 8 weeks after FESS in all 3 groups. At 12 weeks after FESS, the bacterial growth prevalence rates in patients who took a 4-week course of amoxicillin after FESS was significantly higher than those before FESS $(p=0.002)$, but the prevalence rates of bacterial culture growth of $\mathrm{CHM}$ and placebo groups were not significantly different from those before FESS ( $p=0.525$ and 1 , respectively). The prevalence rates of bacterial growth at 12 weeks after FESS in patients who took a 4-week course of amoxicillin after FESS was also significantly higher than those of CHM and placebo groups $(p=0.043$ and 0.016 , respectively), but the bacterial prevalence rates at 12 weeks after FESS were not significantly different between CHM and placebo groups ( $\mathrm{p}$ $=0.775$ ).

The bacteriologies of the 3 groups before and after FESS are shown in Tables 2-4. The growth of Staphylococcus aureus increased in all 3 groups either at 8 or 12 weeks after FESS. Although patients in amoxicillin group took a 4-week course of amoxicillin after FESS,

Table 2. Bacteriology of Chinese herbal medicine group ( $\left.46^{\#}\right)$.

\begin{tabular}{|c|c|c|c|}
\hline & Pre-FESS & $\begin{array}{l}\text { 2M after } \\
\text { FESS }\end{array}$ & $\begin{array}{c}\text { 3M after } \\
\text { FESS }\end{array}$ \\
\hline Species & & $\begin{array}{c}\text { No. of } \\
\text { isolates }\end{array}$ & \\
\hline \multicolumn{4}{|l|}{ Aerobic and facultative bacteria } \\
\hline \multicolumn{4}{|l|}{ Gram-positive } \\
\hline Coagulase-negative staphylococci & 7 & 2 & 2 \\
\hline Staphylococcus aureus & 4 & 8 & 8 \\
\hline Streptococcus pneumoniae & 2 & 2 & 1 \\
\hline Corynebacterium spp. & 2 & & \\
\hline Lactobacillus sp. & 2 & & \\
\hline \multicolumn{4}{|l|}{ Gram-negative } \\
\hline Klebsiella oxytoca & & 1 & \\
\hline Enterobacter aerogenes & 1 & & 2 \\
\hline Citobacter koseri & 1 & 2 & \\
\hline Nonfermentative G(-) bacillus & & 2 & \\
\hline Total aerobic and facultative bacteria & 19 & 17 & 13 \\
\hline \multicolumn{4}{|l|}{ Anaerobic bacteria } \\
\hline \multicolumn{4}{|l|}{ Gram-positive } \\
\hline Propionibacterium acnes & 3 & 4 & 4 \\
\hline Total anaerobic bacteria & 3 & 4 & 4 \\
\hline Total bacterial isolates & 22 & 21 & 17 \\
\hline
\end{tabular}

FESS: functional endoscopic sinus surgery; ${ }^{\#}$ Number of specimens.
Table 3. Bacteriology of amoxicillin group $\left(54^{\#}\right)$.

\begin{tabular}{|c|c|c|c|}
\hline & Pre-FESS & $\begin{array}{c}2 \mathrm{M} \text { after } \\
\text { FESS }\end{array}$ & $\begin{array}{c}3 \mathrm{M} \text { after } \\
\text { FESS }\end{array}$ \\
\hline Species & & $\begin{array}{l}\text { No. of } \\
\text { isolates }\end{array}$ & \\
\hline \multicolumn{4}{|l|}{ Aerobic and facultative bacteria } \\
\hline \multicolumn{4}{|l|}{ Gram-positive } \\
\hline Coagulase-negative staphylococci & 5 & 3 & 6 \\
\hline Staphylococcus aureus & & 11 & 8 \\
\hline Streptococcus pneumoniae & 2 & 4 & 5 \\
\hline Corynebacterium spp. & 3 & 2 & 2 \\
\hline \multicolumn{4}{|l|}{ Gram-negative } \\
\hline Klebsiella pneumoniae & 3 & 5 & 5 \\
\hline Haemophilus influenzae & 4 & 1 & 1 \\
\hline Pseudomonas aeruginosa & & & 1 \\
\hline Pseudomonas stutzeri & & 1 & 1 \\
\hline Citobacter koseri & & 2 & 3 \\
\hline Nonfermentative G(-) bacillus & 1 & & 1 \\
\hline Pantoea agglomerans & 1 & & \\
\hline Total aerobic and facultative bacteria & 19 & 29 & 33 \\
\hline \multicolumn{4}{|l|}{ Anaerobic bacteria } \\
\hline \multicolumn{4}{|l|}{ Gram-positive } \\
\hline Propionibacterium acnes & & 4 & 2 \\
\hline Propionibacterium spp. & & 1 & \\
\hline \multicolumn{4}{|l|}{ Gram-negative } \\
\hline Prevotella corporis & & & 2 \\
\hline Total anaerobic bacteria & & 5 & 4 \\
\hline Total bacterial isolates & 19 & 34 & 37 \\
\hline
\end{tabular}

FESS: functional endoscopic sinus surgery; "Number of specimens.

only 1 of 11 isolates of Staphylococcus aureus at 8 weeks after FESS was resistant to methicillin, and none of 8 isolates of Staphylococcus aureus at 12 weeks after FESS was resistant to methicillin.

\section{Discussion}

Antibiotics have been used routinely after FESS [3]. Although few studies have reported the bacteriology of CRS after FESS [9], broad-spectrum antibiotics have been recommended for the medical management of CRS because of a diverse range of bacteria involved in CRS. Recently, macrolides have also been used to treat CRS, mainly based on their immunomodulatory effect instead of antibiotic activity [10]. However, the use of broadspectrum antibiotics or low-dose macrolides has theoretical risks to develop bacterial resistance both in the individual patient and in the community [11]. Therefore, in this study, we chose Jia Wei Cang Er San to manage post-FESS patients because it has been widely used to 
Table 4. Bacteriology of placebo group (38*).

\begin{tabular}{|c|c|c|c|}
\hline & Pre-FESS & $\begin{array}{c}\text { 2M after } \\
\text { FESS }\end{array}$ & $\begin{array}{c}3 \mathrm{M} \text { after } \\
\text { FESS }\end{array}$ \\
\hline Species & & $\begin{array}{l}\text { No. of } \\
\text { isolates }\end{array}$ & \\
\hline
\end{tabular}

Aerobic and facultative bacteria

Gram-positive

\begin{tabular}{lccc} 
Coagulase-negative staphylococci & 2 & 1 & \\
Staphylococcus aureus & & 8 & 3 \\
Streptococcus pneumoniae & 1 & 1 & 2 \\
Bacillus spp. & 1 & & \\
Gram-negative & & & \\
Klebsiella pneumoniae & 2 & 2 & 1 \\
Klebsiella ozanenae & 3 & 1 & \\
Enterobacter aerogenes & & & 1 \\
Acinetobacter baumannii & 2 & 1 & 1 \\
Nonfermentative G(-) bacillus & 11 & 14 & 8 \\
Total aerobic and facultative bacteria & & & \\
Anaerobic bacteria & & & \\
Gram-positive & 5 & 3 & 3 \\
Propionibacterium acnes & & & 1 \\
Propionibacterium spp. & & & \\
Gram-negative & & 5 & 5 \\
Veillonella spp. & 16 & 17 & 13 \\
Total anaerobic bacteria & & \\
Total bacterial isolates & & & \\
\hline
\end{tabular}

FESS: functional endoscopic sinus surgery; ${ }^{*}$ Number of specimens.

treat rhinosinusitis in traditional Chinese Medicine due to its putative immunomodulatory effect $[6,12]$.

Our results showed that endoscopic scores decreased significantly at 8 and 12 weeks after FESS in all groups, but there were no significant differences in endoscopic scores among the 3 groups at 12 weeks after FESS. This indicates that postoperative CHM or amoxicillin treatment did not add additional effect in post-FESS improvement.

However, when the effect of postoperative CHM or amoxicillin treatment on the bacteriology was evaluated, the prevalence rates of positive bacterial culture increased at 8 weeks after FESS in all groups, but decreased at 12 weeks after FESS in patients who took Jia Wei Cang Er San or placebo. On the other hand, the prevalence rates of positive bacterial culture in those who took amoxicillin still increased at 12 weeks after FESS. This suggested that postoperative amoxicillin treatment did not reduce bacterial growth but, paradoxically, prolonged their growth. In this study, we used Jia Wei Cang Er San to compare with amoxicillin to evaluate the influence on the bacteriology after FESS. Although one of its components, Angelica dahurica, has been postulated to exert antibiotic effects, it is believed that Jia Wei Cang Er San does not have strong an antibiotic effect as amoxicillin. Our results showed that the influence of Jia Wei Cang Er San on the bacteriology after FESS was almost the same as that of placebo.

\section{Conclusion}

When antibiotics have been used routinely to treat CRS, the use of antibiotics has theoretical risks to develop bacterial resistance. Although the effect of Jia Wei Cang Er San in the treatment of CRS needs further investigation, our study showed that it did not induce bacterial growth after FESS as amoxicillin.

\section{Acknowledgements}

We are grateful to Dr. Yi-Chang Su, School of Chinese Medicine, China Medical University, Taichung, Taiwan, for his assistance in this study. We are also grateful to the Biostatistics Task Force, Taichung Veterans General Hospital, Taichung, Taiwan, for their statistical assistance. This study was supported by the research grant CCMP96RD-016 from the Committee on Chinese Medicine and Pharmacy, Department of Health, Excusive Yuan, Taiwan.

\section{REFERENCES}

[1] E. R. Thaler, "ER: Postoperative Care after Endoscopic Sinus Surgery," Archives of Otolaryngology_Head \& Neck Surgery, Vol. 28, No. 10, 2002, pp. 1204-1206.

[2] M. Jorissen, "Postoperative Care Following Endoscopic Sinus Surgery," Rhinology, Vol. 42, No. 3, 2004, pp. 114120.

[3] S. V. Fernandes, "Postoperative Care in Functional Endoscopic Sinus Surgery?" Laryngoscope, Vol. 109, No. 6, 1999, pp. 945-948. doi:10.1097/00005537-199906000-00020

[4] R. S. Jiang, K. L. Liang, K. Y. Yang, J. Y. Shiao, et al., "Postoperative Antibiotic Care after Functional Endoscopic Sinus Surgery," American Journal of Rhonology, Vol. 22, No. 6, 2008, pp. 608-612.

[5] Y. Zhao, C. A. van Hasselt, J. K. Woo, et al., "Effect of a Chinese Herbal Formula, Shi-Bi-Lin, on an Experimental Model of Allergic Rhinitis," Annals Allergy, Asthma \& Immunology, Vol. 96, No. 6, 2006, pp. 844-850. doi:10.1016/S1081-1206(10)61348-8

[6] Y. Zhao, K. S. Woo, K. H. Ma, et al., "Treatment of Perennial Allergic Rhinitis using Shi-Bi-Lin, a Chinese Herbal Formula," Journal of Ethnopharmacology, Vol. 122, No. 1, 2009, pp. 100-105. doi:10.1016/j.jep.2008.12.005

[7] D. Lechner, M. Stavri, M. Oluwatuyi, R. Pereda-Miranda and S. Gibbons, "The Anti-Staphylococcal Activity of Angelica Dahurica (Bai Zhi),” Phytochemistry, Vol. 65, No. 3, 2004, pp. 331-335. 
doi:10.1016/j.phytochem.2003.11.010

[8] V. J. Lund and I. S. Mackay, "Staging in Rhinosinusitis," Rhinology, Vol. 31, No. 4, 1997, pp. 183-184.

[9] N. Bhattacharyya and K. J. Kepnes, "The Microbiology of Recurrent Rhinosinusitis after Endoscopic Sinus Surgery," Archives of Otolaryngology-Head \& Neck Surgery, Vol. 125, No. 10, 1999, pp. 1117-1120.

[10] M. Y. Desrosiers and S. J. Kilty, "Treatment Alternatives for Chronic Rhinosinusitis Persisting after FESS: What to Do When Antibiotics, Steroids and Surgery Fail," Rhi- nology, Vol. 46, No. 1, 2008, pp. 3-14.

[11] Y. Y. Kung, Y. C. Chen, S. J. Hwang, T. J. Chen and F. P. Chen, "The Prescriptions Frequencies and Patterns of Chinese Herbal Medicine for Allergic Rhinitis in Taiwan," Allergy, Vol. 61, No. 11, 2006, pp. 1316-1318. doi:10.1111/j.1398-9995.2006.01152.x

[12] Z. M. Soler and T. L. Smith, "What is the Role of Longterm Macrolide Therapy in the Treatment of Recalcitrant Chronic Rhinosinusitis," Laryngoscope, Vol. 119, No. 11, 2009, pp. 2083-2084. doi:10.1002/lary.20739 